\title{
eJRIEPS
}

Ejournal de la recherche sur l'intervention en éducation physique et sport

$23 \mid 2011$

Varia

\section{La prise en compte de l'adversité comme objet d'entraînement en Volley-ball : une recherche technologique}

\section{Christine Amans-Passaga}

\section{(2) OpenEdition}

1 Journals

\section{Édition électronique}

URL : http://journals.openedition.org/ejrieps/4552

DOI : $10.4000 /$ ejrieps. 4552

ISSN : 2105-0821

Éditeur

ELLIADD

\section{Référence électronique}

Christine Amans-Passaga, "La prise en compte de l'adversité comme objet d'entraînement en Volleyball : une recherche technologique », eJRIEPS [En ligne], 23 | 2011, mis en ligne le 01 avril 2011, consulté le 21 février 2020. URL : http://journals.openedition.org/ejrieps/4552 ; DOI : 10.4000/ejrieps. 4552

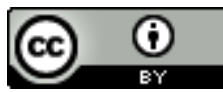

La revue eJRIEPS est mise à disposition selon les termes de la Creative Commons Attribution 4.0 International License. 
La prise en compte de l'adversité comme objet d'entraînement en Volley-ball : une recherche technologique

Christine Amans-Passaga

Département STAPS Université Jean François Champollion Albi Site de Rodez, UMR EFTS Université, Toulouse, France

Résumé

Le présent article a pour vocation de revenir sur une étude réalisée dans le cadre d'une thèse de doctorat STAPS, en Didactique des APS (Amans-Passaga, 1997). Ce travail s'était donné pour objet la question des contenus d'entraînement de joueuses de Volleyball inscrites dans un championnat régional fédéral. Il s'agit ici d'une part de résumer l'essence de cette étude et d'autre part de discuter relativement à la démarche de recherche qui est de type technologique. En conséquence la première partie de cet article présentera la recherche à travers son objet précis, l'ancrage théorique, la démarche choisie pour conduire l'étude et les résultats qu'elle a générés. La deuxième partie discutera son appartenance au champ des recherches technologiques relatives à l'entraînement et à la compétition en sport.

\section{Introduction}

Dans le champ des Sciences de l'intervention, le domaine des études scientifiques sur l'entraînement en sports collectifs s'est étoffé ces dernières années. II s'agit de travaux qui analysent les pratiques d'entraînement, de managérat, de compétition afin de mieux cerner l'intervention et afin d'optimiser l'action des entraîneurs en les dotant de nouvelles connaissances technologiques et didactiques. Certaines études, dont la présente, se donnent pour objet de tester et de valider des procédures d'entraînement originales. La recherche dont il est question dans cet article concerne l'entraînement en volley-ball réenvisagé sous l'éclairage des connaissances issues du courant anglo-saxon nommé «Teaching Games for Understanding» (TGFU) - enseignement des jeux par la compréhension - et des travaux de Bouthier (1988, 1989) relatifs à la comparaison de modèles pédagogiques de l'enseignement des sports collectifs. Elle consiste en une étude de cas, prenant en charge une problématique de terrain, qui fût réalisée dans un contexte réel d'intervention avec une équipe de joueuses de volley-ball de niveau régional. Le choix fut fait de conduire l'étude selon une méthode d'essai - évaluation consistant à concevoir 


\section{eJRIEPS 23 avril 2011}

un protocole de formation, à le tester et à l'évaluer en contexte. La description de certaines des caractéristiques de cette étude au plan méthodologique en particulier, devrait permettre d'illustrer les études technologiques en APSA.

\section{Retour sur l'objet, la démarche et les résultats de la recherche}

\section{1. Objet}

Au cœur de l'étude développée dans le cadre d'une thèse de didactique des APSA (Amans-Passaga, 1997), se trouve une conception de l'enseignement du volley-ball valorisant le développement de compétences tactiques dès le début de la formation. L'expérience support conduite par l'entraîneur (qui est aussi le chercheur) consiste en la formation d'une équipe de joueuses de volley-ball de niveau régional à partir d'un travail systématique sur l'observation des spécificités de l'adversaire, pour la prise en compte de celles-ci dans les décisions tactiques. Cette formation concerne d'une part la prise d'information cumulée sur les spécificités adverses (système de jeu, placements collectifs, particularités individuelles des joueuses) et d'autre part le rôle de ces informations dans les prises de décision. Elle s'actualise à travers des situations de formation insérant des contenus centrés sur le décryptage de signaux, d'indices relatifs aux dispositifs adverses, proposées dans les séquences d'entraînement au fil de la saison. Cette option de formation est en phase avec certains modèles d'analyse des sports collectifs évoqués cidessus mais en relative rupture d'une part avec les considérations du milieu fédéral concernant la formation des joueurs de ce niveau et d'autre part avec les pratiques d'entraînement en usage.

\section{2. Cadre théorique}

Les fondements théoriques à l'origine de l'étude et du choix de son objet se trouvent donc dans les approches des sports collectifs dites «par la compréhension » (Bunker \& Thorpe, 1983), identifiées sous le vocable TGFU (Teaching Games for Understanding). Ce courant d'analyse des sports collectifs préconise le développement prioritaire des compétences cognitives du joueur (analyse des situations, choix des solutions, mémorisation des évènements du jeu) dans des situations incluant un rapport d'opposition. En France, les travaux de Bouthier (1988, 1989) et d'Eloi (2000) comparent différents modèles pédagogiques d'enseignement des sports collectifs et montrent d'une part toute la pertinence de modèles qui se centrent sur la formation aux prises de décisions tactiques et d'autre part tout l'intérêt des situations d'opposition, de jeu à effectif réduit, dans l'entraînement. D'autres auteurs aboutissent à des conclusions très proches (Grehaigne \& Gotbout, 1995, Gréhaigne, Godbout \& Mahut, N, 1999) en montrant en particulier que 


\section{eJRIEPS 23 avril 2011}

l'efficacité du joueur passe par une certaine autonomie de sa part en match puisque lui incombe en dernier ressort, au-delà des consignes du manager, la responsabilité de ses choix.

\section{3. Démarche et méthodologie}

A l'origine du programme de recherche ici décrit, se trouve un état des lieux qui révèle la faiblesse des joueuses relativement à la prise en compte de l'adversaire. Cette faiblesse a pour conséquence un jeu stéréotypé quelle que soit l'équipe affrontée. Cet état de fait a amené l'entraîneur à mettre en œuvre un essai-évaluation qui consiste en la définition d'un programme de travail autour du contenu : "s'informer sur ce qu'est et ce que fait l'adversaire pour aider à la décision » et dans son introduction sur une demi-saison de championnat en ciblant prioritairement le secteur de jeu service-réception. L'étape finale consistant à évaluer les effets en termes d'amélioration des compétences visées et en terme de performance sportive.

Le déroulement du programme s'est, pour les besoins de la recherche, accompagnée d'un recueil de données qualitatives et quantitatives armé d'outils spécifiques (entretiens, observations, quantifications d'actions) construits pour la circonstance. II visait à objectiver la situation et ce d'autant plus que, comme dit précédemment, une même personne compilait les fonctions d'entraîneur et de chercheur.

\section{4. Résultats}

Ces outils ont en l'occurrence permis une objectivation des transformations des compétences des joueuses : des analyses statistiques portant sur les réponses à des questionnaires de fin de match (vérifiant le décryptage des spécificités de l'adversaire), sur l'efficacité des services et des réceptions pendant les matches et sur la corrélation entre ces deux points révèlent des progrès chez les joueuses au regard de la compétence visée : caractériser un adversaire et en tirer parti pour décider. Des analyses qualitatives sur la répartition spatiale des services effectués par nos joueuses sur le terrain adverse montrent une tendance à une meilleure exploitation de l'occupation de cet espace par les réceptionneurs.

La formation à la détection des spécificités de l'adversaire dans le secteur serviceréception s'est donc avérée être un objet d'entraînement pertinent pour le niveau concerné. Elle a contribué à inscrire les joueuses dans une approche plus tactique de l'opposition en match basée sur l'utilisation d'indicateurs autres que ceux prélevés dans l'instant. Les contenus de formation proposés ont également eu des effets positifs sur l'efficacité en match (résultats des rencontres). Cependant, on a conclu que cette formation est exigeante en temps (elle s'était étalée sur une demi- saison de championnat) 


\section{eJRIEPS 23 avril 2011}

et demande à être poursuivie pour être renforcée : en effet, les observations faites lors d'un match difficile, face à un adversaire d'un niveau supérieur, ont révélé le retour des joueuses à des conduites moins évoluées, plus centrées sur leur propre jeu et sur la prise en compte d'informations ponctuelles dès lors qu'elles se trouvaient en situation de plus grande crise.

Au terme de l'étude, on a donc conclu que le décryptage des spécificités de l'adversaire constitue une compétence envisageable à ce niveau de jeu. Cela peut contribuer à l'optimisation des résultats, installer les joueuses dans une perspective de jeu à plus haut niveau, introduire et contextualiser les apprentissages techniques. Des conclusions qui corroborent celles des études citées en introduction relativement à l'opportunité du développement précoce des compétences tactiques (Amans-Passaga, Amade-Escot \& Bouthier, 2000).

Après une présentation rapide de ce travail, venons en à présent à l'objet propre de cet article qui consiste à caractériser la nature de la recherche que nous venons de résumer et à montrer son inscription dans la catégorie des études technologiques.

\section{Analyse de la dimension technologique de cette recherche}

Nous procèderons ici en présentant certaines des caractéristiques propres aux études technologiques et en les illustrant par notre étude.

3. 1. Les recherches technologiques poursuivent une finalité d'optimisation de l'intervention à partir de l'investigation d'un système complexe

Dès 1973, dans l'Encyclopédia Universalis, Guillerme (1973) définissait la technologie comme «l'usage rationnel des techniques dans le cadre de la conception, la mise en œuvre et la réalisation d'un projet d'intervention sur le réel destiné à transformer celui-ci en respectant sa complexité ». L'approche technologique se veut en effet une démarche qui permet d'approcher des situations naturelles en les investiguant dans leur complexité, sans réduction ni simplification. Parce que la technologie est «une science humaine des techniques» (Haudricourt, 1987) son objet est de formaliser les techniques des acteurs, pratiquants et intervenants dans leur contexte. De fait elle autorise la convocation d'un ensemble composite de connaissances scientifiques et professionnelles, d'intuitions, de connaissances empiriques, d'expériences personnelles.

Dans la présente recherche, il était en effet question d'utiliser une démarche scientifique pour résoudre une question de terrain, celle d'un entraîneur en proie aux difficultés de ses joueuses au niveau de leurs compétences tactiques. Ainsi, le point de départ de la recherche résidait dans le repérage de cette compétence d'observation déficiente. Celle-ci 


\section{eJRIEPS 23 avril 2011}

justifiait la conception d'un programme de formation autour de la prise en compte des adversaires, sa mise à l'essai effective sur une demi-saison de championnat et la mesure des effets produits sur les compétences et les performances des joueuses. Le tout soustendu par la finalité de transformation, d'optimisation et d'efficacité de l'entraînement. La définition de cet objet avait émergé de la confrontation de différents types de connaissances : des préalables théoriques relatifs à la question de la prise d'information dans un modèle cognitif du fonctionnement du sujet, des considérations expertes sur l'enseignement des sports collectifs, et des intuitions individuellement construites au fil de notre expérience d'entraîneur.

3. 2. Les recherches technologiques supposent une démarche longitudinale de type conception, essai, évaluation.

La démarche technologique s'actualise dans différentes étapes : la première consiste à analyser le contexte d'où sont issues les préoccupations confiées à la recherche. Vient ensuite le recensement des connaissances théoriques, scientifiques, professionnelles pouvant éclairer la question de départ. La construction de l'outil, du projet de formation destiné à améliorer le fonctionnement du système technique constitue l'étape suivant. Vient ensuite la mise en œuvre effective du projet qui nécessite immanquablement des ajustements par rapport au modèle initialement conçu. La phase ultime de la démarche technologique concerne l'évaluation du projet au regard des effets en terme d'optimisation du système technique. Ces étapes ici présentées comme successives sont en pratique temporellement imbriquées et supposent une mise en œuvre sur une certaine durée, nécessaire à la transformation d'un système humain.

Dans notre recherche, nous avons mobilisé des connaissances scientifiques relatives au traitement de l'information, des connaissances expertes empruntées aux spécialistes de volley-ball relativement à l'intérêt de la formation des compétences tactiques. Sur cette base, nous avons conçu un programme de formation composé de situations de travail insérant le thème de la formation au décryptage et à l'utilisation d'informations adverses. Ce programme a ensuite été mis en œuvre dans une demi-saison de championnat, le temps de la formation dictant celui de la recherche. L'évaluation a été possible grâce à une méthodologie taillée sur mesure assortie d'une analyse vidéo. Elle a consisté dans le relevé et le traitement croisé d'une part de données quantitatives sur l'efficacité dans le secteur service / réception au cours des différents matches et d'autre part de données qualitatives sur les informations prélevées par les joueuses sur l'équipe adverse grâce à des questionnaires d'après matches. Pour exemple, la mémorisation du vécu individuel au cours des matchs fut évaluée à travers l'investigation de deux catégories d'informations : 


\section{eJRIEPS 23 avril 2011}

la connaissance acquise sur l'adversaire (caractérisation des serveurs adverses, dispositif collectif en réception adverse) et la perception de l'efficacité du joueur lui-même (choix valorisés et effets de ces choix) que nous avons pu confronter aux données réelles grâce aux vidéos des matches.

\section{3. L'étude technologique convoque différents registres de technicité}

A la suite de Martinand (1986), Bouthier \& Durey (1994) ont proposé différents registres de technicité en technologie des APSA : le registre de la maîtrise des techniques sportives ; celui de leur transformation ; le registre de lecture et d'analyse des productions sportives ; enfin, le registre de participation qui renvoie aux différents rôles ou fonctions endossables par les acteurs sportifs. Chacun de ces registres supposant le déploiement d'habiletés, de compétences, d'outils et d'instruments. Concernant notre étude, le registre mobilisé préférentiellement est celui de la transformation puisqu'au centre de nos préoccupations se trouvaient les procédures et les contenus d'entraînement d'un intervenant sportif préoccupé par l'optimisation des conduites perceptivo-décisionnelles de ses joueuses. II s'agissait de mettre ces options de formation à l'épreuve - pour éventuellement les valider tout au moins sur le plan local - dans un système réel. Cependant, sont également convoqués à titre contributoire :

- le registre de maîtrise tout d'abord puisque ce travail investigue la pertinence potentielle d'un facteur d'efficacité en volley-ball donc de maîtrise dans cette activité. Notre étude se propose de vérifier que la prise d'information sur l'adversaire participe à la construction de l'expertise d'un joueur de volley-ball même à ce niveau de jeu ; cette étude contribue donc à une analyse des compétences contributives à la performance en volley-ball à un niveau donné.

- le registre de lecture : la conduite de l'étude supposait de se doter des moyens pour interpréter l'activité des joueuses. Sachant qu'il a fallu objectiver non seulement leurs actions motrices dans le secteur service/réception mais aussi leurs intentions et leurs prises d'informations. Pour cela, nous avons développé des moyens méthodologiques permettant d'accéder à ces intentions, aux prises d'informations effectives, aux effets de ces connaissances sur les décisions. En cela, le couplage de l'étude des points de chute des services sur le terrain adverse à partir des enregistrements vidéos des matchs, et de l'analyse des questionnaires d'après match, a permis d'objectiver les progrès sur le plan de la prise en compte des spécificités adverses.

- Au registre de la participation, la situation particulière choisie dans cette recherche imposait à une même personne d'endosser au même moment des fonctions de chercheur, d'entraîneur et de manager ; compilation de rôles parfois délicate qui a conduit à des choix 


\section{eJRIEPS 23 avril 2011}

et des priorités. Pour illustrer la difficulté de la tâche évoquons le télescopage existant en situation de match entre les fonctions du chercheur qui veut vérifier le relevé d'informations sur les adversaires réellement effectué par ses joueuses et celles du manager qui a intérêt à accompagner voir à dicter les choix tactiques de ses joueuses dans une perspective d'efficacité immédiate et de performance.

3. 4. Les recherches technologiques génèrent la production de connaissances utiles pour l'intervention

L'utilité des recherches technologiques dépasse le plan local et la production de connaissances inhérentes au système investigué. Certes, toute recherche conduite doit avant tout satisfaire la demande des praticiens qui ont confié au(x) chercheur(s) une question de terrain et qui sont légitimement demandeurs de résultats susceptibles de contribuer à améliorer leur efficacité professionnelle. Mais les résultats locaux peuvent également intéresser une communauté d'intervenants sensibilisés au même objet ; de fait le travail de mise à disposition et de diffusion des conclusions fait partie intégrante de l'étude technologique.

Concernant cette diffusion, même s'il y a production d'un outil (le programme de formation bâti pour la circonstance), l'intérêt reste relatif du fait de la difficulté à convaincre un secteur fédéral dont les pratiques de terrain sont encore installées sur une conception plutôt techniciste de l'entraînement. Si intérêt de ce travail il y a au plan large, il se trouve davantage dans le fait qu'il vient corroborer des conclusions proches portées par des auteurs français et étrangers spécialistes des sports collectifs, cités en introduction. Nos conclusions rejoignent les leurs au sujet de l'intérêt d'une formation des compétences tactiques et d'une formation par le jeu dés l'apprentissage. II en va ainsi de l'utilité des recherches de type «études de cas ». La proximité des objets étudiés et la convergence des résultats produits dans des études portant sur des sports de balle différents, avec des publics variés, sont susceptibles de conférer une validité aux orientations théoriques fondamentales qui les sous-tendent.

3. 5. La position originale du chercheur dans les recherches technologiques

Les recherches technologiques en APSA revendiquent de prendre en charge des questions émanant de professionnels pour aider à leur résolution. De fait, elles ne peuvent que reposer sur des collaborations étroites entre d'une part un ou des chercheur(s) fins connaisseurs des systèmes étudiés et ayant le souci des retombées des travaux de recherche sur les systèmes qu'ils étudient; et d'autre part un ou des praticien(s) demandeurs d'une analyse fine, d'une optimisation de leurs actions d'intervention, euxmêmes engagés dans une démarche réflexive et qui de fait deviennent de précieux 


\section{eJRIEPS 23 avril 2011}

collaborateurs de recherche. La collaboration doit d'ailleurs exister aux différentes étapes depuis la phase initiale du projet, lors de la conception et de la mise en œuvre. Elle s'actualise particulièrement dans la réflexion autour des ajustements du processus innovant grâce aux feed-back renvoyés par les utilisateurs, permettant d'optimiser l'outil. De fait, le chercheur doit dans ce type de recherche renoncer à une attitude prescriptive et opter pour des formes originales et innovantes de coopération à partir d'une reconnaissance symétrique des connaissances et compétences des chercheurs et des intervenants comme le développe Desgagné (1998) à propos des recherches collaboratives en éducation.

Dans notre cas, la collaboration a pris une dimension particulière dans la mesure où chercheur et entraîneur se trouvaient réunis en une seule et même personne. Ce qui n'est pas sans conséquence sur l'outillage méthodologique et sur l'exigence de vigilance forte de l'entraîneur-chercheur sur les analyses faites, les conclusions produites, du fait de sa position impliquée et non extérieure.

Concernant l'originalité et la spécificité de ce type de recherche, nous ne pouvons enfin passer sous silence le fait que les joueuses constituent également de vrais partenaires de la recherche et deviennent partie prenante du processus de recherche. Nécessairement informées des options théoriques et des préoccupations de leur entraîneur-chercheur, l'adhésion des joueuses est suggérée, parce qu'elles savent par exemple qu'elles devront à l'issue du match remplir un questionnaire portant sur les spécificités adverses. Elle l'est aussi chez les remplaçantes dont on voit la conduite se modifier sur le banc de touche durant les matchs : leur passivité habituelle se change, pour les mêmes raisons, en démarche active d'observation de l'adversaire et en distribution d'informations pertinentes à leurs partenaires. Dès lors on peut dire que le questionnaire de fin de match, construit en tant qu'outil de recherche, devient outil de formation. Les «perturbations » introduites par la recherche donnent finalement à la formation une valeur ajoutée.

\section{Conclusion}

L'objectif de cette contribution était double : il s'agissait de présenter synthétiquement une recherche portant sur l'entraînement en volley-ball et de montrer, à partir de certaines caractéristiques du travail, son inscription dans l'approche technologique en APSA (Mouchet, Amans-Passaga \& Gréhaigne, 2010). Cette étude illustre également le fait qu'une recherche de type didactique centrée sur les contenus placés au cœur d'un processus d'intervention peut être conduite selon une démarche technologique. Enfin, elle confirme s'il en était besoin que le choix des objets et des démarches de recherche n'est 


\section{eJRIEPS 23 avril 2011}

pas anodin pour le chercheur en intervention en APSA qui endosse aussi le plus souvent, dans une institution de formation, la posture de formateur. Pour notre part le choix de l'objet et des questions de recherche était en lien étroit avec les responsabilités assumées d'une part dans le secteur de l'entraînement en volley-ball et d'autre part dans la formation d'intervenants en APSA, dans le champ de la technologie des sports collectifs. Ainsi, l'intrusion de la recherche dans l'action d'intervention quotidienne telle que pratiquée dans la démarche technologique et telle qu'illustrée ici, en associant les acteurs, se révèle une voie possible pour faire évoluer les pratiques d'intervention.

\section{Bibliographie}

Amans-Passaga, C. (1997). Prise en compte de l'adversité en Volley-Ball : essai évaluation d'un objet d'entraînement. Thèse de Doctorat en Didactique des Disciplines Option APS, Université Paul Sabatier.

Amans-Passaga, C., Amade-Escot, C., Bouthier, D. (2000). La prise en compte de l'adversaire comme objet d'entraînement en volley-ball. Contribution aux recherches sur la formation tactique en sports collectifs. Sciences et Motricité, 41, 86-94.

Bouthier, D. (1988). Contribution de la psychologie à la problématique de formation des actions sportives collectives. Thèse de doctorat. EPHE. Université Paris V, Paris.

Bouthier, D. (1989). Les conditions cognitives de la formation d'actions sportives collectives. Le Travail Humain, 52, 2, 175-182.

Bouthier, D., Durey, A. (1994). Technologie des APS. Impulsion, 1, 95-120.

Bunker D., Thorpe, R. (1983). A model for the teaching of games in secondary schools. Bulletin of Physical Education, Vol.19, 1.

Desgagné, S. (1998). La position du chercheur en recherche collaborative : illustration d'une démarche de médiation entre culture universitaire et culture scolaire. Recherches Qualitatives, 18, 77-105.

Eloi, S., (2000), Représentations mentales et acquisition d'habiletés tactiques en volleyball : Effets comparés de deux cycles d'enseignement. Science et motricité, 40, 22-31.

Gréhaigne, J.F., Gotbout, P. (1995). Tactical knowledge in team sports from a constructivist and cognitivist perspective. Quest, 45, 490-505.

Gréhaigne, J.F., Godbout, P., Mahut, N. (1999). L'enseignement des jeux par la compréhension : une revue de question ». STAPS, 48, 81-93. 


\section{eJRIEPS 23 avril 2011}

Guillerme, J., (1973). Technologie. Encyclopaëdia Universalis, 15, 820-823.

Haudricourt, G., (1987). Technologie science humaine, Paris, Maison des Sciences de l'Homme.

Martinand, J.L., (1986). Connaître et comprendre la matière. Berne : Peter Lang.

Mouchet, A., Amans-Passaga, C. , Grehaigne, J.F. (2010). L'approche technologique. In Musard, M., Loquet, M. \& Carlier, G. (Eds.). Sciences de l'intervention en EPS et en sport, Résultats de recherche et fondements théoriques. (pp. 201-222). Paris : revue EPS. 